\title{
Heating of Pickup and Solar Wind Ions at Jupiter's Bow Shock
}

\author{
George Gloeckler*†, Johannes Geiss", and Lennard A. Fisk ${ }^{\dagger}$ \\ * Department of Physics and IPST, University of Maryland, College Park MD 20742, USA \\ ${ }^{\dagger}$ Department of Atmospheric, Oceanic, and Space Sciences, University of Michigan, \\ Ann Arbor MI 48109, USA \\ International Space Science Institute, CH-3012 Bern, Switzerland
}

\begin{abstract}
Interstellar pickup ions are dynamically important in the outer heliosphere where they mass-load and heat the solar wind. Some of these pickup ions are transformed into energetic neutral atoms (ENAs) by charge exchange with the residual cold interstellar gas that is the primary constituent of the outer heliosphere. The most detailed measurements of interstellar pickup ions in the heliosphere are currently available only between $\sim 1$ and $\sim 5$ AU. Among the most interesting and least expected observations are those of ubiquitous suprathermal tails on the distribution of pickup and solar wind protons and all heavier ions that can be measured. Here we report new measurements of solar wind proton and alpha particle distributions and of pickup $\mathrm{He}^{+}$ spectra upstream and downstream of Jupiter's bow shock. We find that in the magnetosheath, $27 \%$ of the total pickup $\mathrm{H}^{+}$density is in the tail portion of the distribution, compared to only $0.4 \%$ in the upstream spectrum. For $\mathrm{He}^{+}$the entire core distribution is apparently heated in crossing the shock. These results have important implications for particle acceleration at the heliospheric termination shock, and for predicting the fluxes of energetic neutral atoms in the inner heliosphere produced from solar wind and pickup ions heated and accelerated at the termination shock.
\end{abstract}

\section{INTRODUCTION}

Interstellar pickup ions, predicted to exist deep inside the heliosphere [1,2] long before the launches of the Voyager spacecraft, were finally observed many years thereafter $[3,4,5]$. A new extended source of pickup ions, the so-called 'Inner Source', was discovered [6,7] and studied [7,8] using data from the Solar Wind Ion Composition Spectrometer (SWICS) on Ulysses [9]. Detailed measurements of pickup ions from both of these extended sources, primarily with SWICS on Ulysses at 1.4 to $5.4 \mathrm{AU}$ and ACE at $1 \mathrm{AU}$, have given us new insights on the interactions of these hightemperature ions with the solar wind. Using this information it is possible to extrapolate pickup ion observations in the inner heliosphere to distances beyond $5 \mathrm{AU}$, and thus infer the likely characteristics of pickup ions in the distant heliosphere where, as it turned out, Voyagers are not equipped to make these measurements. It is also possible to deduce the chemical and isotopic abundances of the neutral component of the interstellar gas from observations of pickup ions deep inside the heliosphere.

CP719, Physics of the Outer Heliosphere: Third International IGPP Conference,

edited by V. Florinski, N. V. Pogorelov, and G. P. Zank

(C) 2004 American Institute of Physics 0-7354-0199-3/04/\$22.00 
In the outer heliosphere pickup ions are dynamically important. They mass-load and heat the solar wind and cause it to slow down. Production of additional pickup ions by electron-impact ionization of interstellar neutrals in the vicinity of the termination shock can cause additional slowing of the solar wind, as shown in Fig. 1, if there is significant heating and acceleration of solar wind electrons upstream of the termination shock. Finally, pickup ions with sufficient energy to move toward the Sun will produce energetic neutral atoms (ENAs) that can be observed even at $1 \mathrm{AU}$ as described elsewhere in these Proceedings [10].

\section{ACCELERATION OF PICKUP AND SOLAR WIND IONS BY A STANDING SHOCK}

While it will not be possible to observe the heating of pickup and the solar wind ions by the termination shock with Voyager 1, Ulysses is properly instrumented to study these processes at the bow shock of Jupiter. The Jovian bow shock is a reasonable analogue to the termination shock and one would expect that both shocks would heat and accelerate ions in a similar fashion.

In Fig. 2 are shown the solar wind speed and the counting rates of protons and penetrating particles measured by SWICS on Ulysses during the inbound pass in February 1992. The bow shock location is clearly marked by the dropout of the solar wind speed and the sharp decrease in the proton rate. A rapid increase in the penetrating particles rate and a further drop in the proton rate indicate the location of the magnetopause. The times of bow shock and magnetopause crossings agree with corresponding times deduced from magnetic field observations [11].

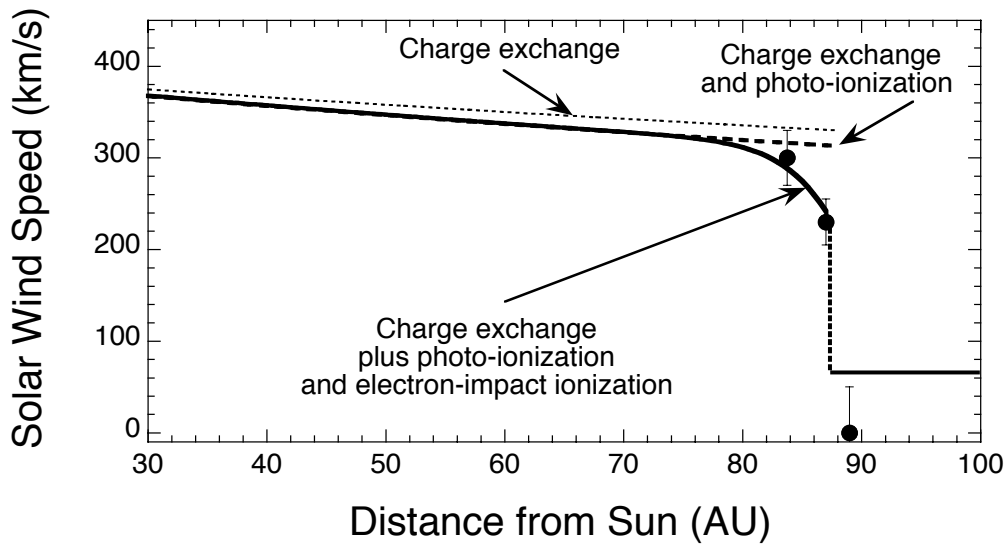

FIGURE 1. Decrease of solar wind speed with distance from the Sun. Filled circles are solar wind speeds inferred [19] from anisotropy measurements of low energy ions on Voyager 1. The rapid slowdown of the solar wind (bold solid curve) may be caused by additional mass loading from pickup ions produced from interstellar neutrals by electron-impact ionization from an assumed source of hot electrons near the termination shock. The predicted slow-downs without electron-impact ionization (dotted and dashed curves) are inconsistent with these Voyager observations. 


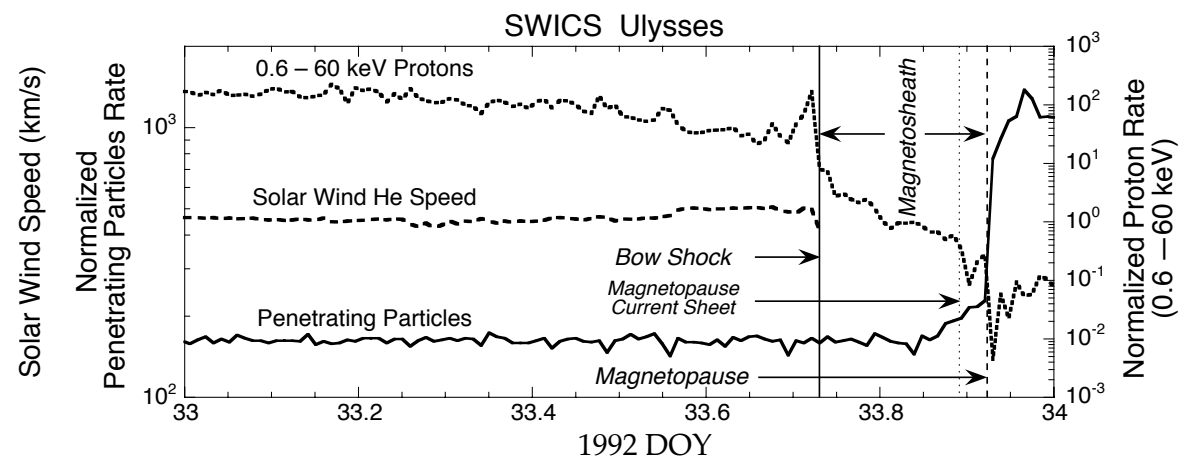

FIGURE 2. Solar wind speed and counting rates of $0.6-60 \mathrm{keV}$ protons and penetrating particles during the inbound pass of Ulysses through the Jovian magnetosphere on 2 February 1992 showing the locations of the bow shock and magnetopause. Behind the bow shock the core solar wind $\mathrm{H}^{+}$falls below the $0.6 \mathrm{keV} / \mathrm{e}$ energy limit of SWICS causing the sudden drop in the counting rate of protons.

Differential energy spectra for $\mathrm{H}^{+}, \mathrm{He}^{++}$and $\mathrm{He}^{+}$, shown in Fig. 3, were obtained both in the upstream region in the solar wind and in the magnetosheath, downstream of the bow shock. Solar wind proton and alpha particle peaks in the spectra upstream of the bow shock (Fig. 3a) are below the energy limit of SWICS in the magnetosheath, and thus not visible in the spectra shown in Fig. 3b. Pickup $\mathrm{He}^{+}$has the usual cutoff in the upstream region. This cutoff is not seen downstream of the bow shock. Finally, while the intensity in the suprathermal tail portion of the $\mathrm{H}^{+}$and $\mathrm{He}^{++}$spectra is substantially higher in the downstream compared to the upstream region, the intensity of pickup $\mathrm{He}^{+}$between $\sim 0.4$ and $\sim 7 \mathrm{keV} /$ nuc is actually lower in the magnetosheath compared to its value in the solar wind.

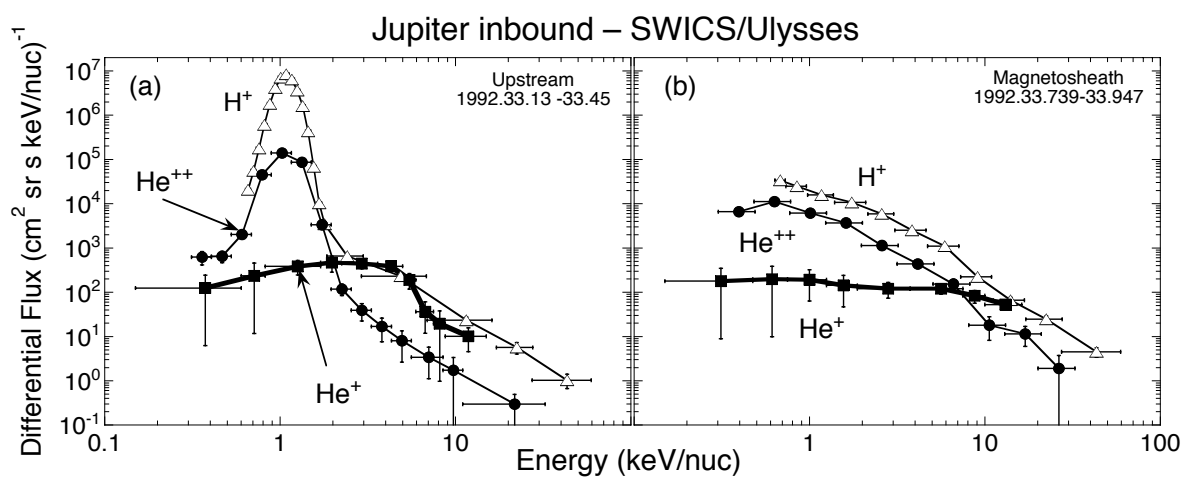

FIGURE 3. Differential energy spectra of $\mathrm{H}^{+}, \mathrm{He}^{++}$and $\mathrm{He}^{+}$, averaged over the indicated time intervals in regions (a) upstream (solar wind) and (b) downstream (magnetosheath) of the Jovian bow shock. 


\section{PICKUP ION DISTRIBUTIONS DOWNSTREAM OF THE SHOCK}

We first consider the transformation of the proton distribution in crossing the bow shock. In Fig. 4a the proton spectrum, measured in the energy range of SWICS (shaded portion) in the upstream region (filled circles), is well represented by the sum of two convected isotropic kappa functions (CIKFs). The convection speed of each is the $456 \mathrm{~km} / \mathrm{s}$ solar wind speed. The parameter values for the number density, $n$, thermal speed, $V_{t h}$, and $\kappa$ for the core and tail distributions are indicated in the figure.

In the downstream (magnetosheath) region the solar wind peak of the core distribution is no longer visible, but the intensity in the tail is about a factor of 8 higher than in the tail of the solar wind distribution upstream of the shock. In the magnetosheath the average solar wind electron density $[12,13]$, and thus also the average magnetosheath solar wind proton density, is $\sim 0.146 \mathrm{~cm}^{-3}$ which is 2.43 times the upstream proton density. The convective velocity, $\boldsymbol{V}$, in the downstream region has been determined from solar wind electron measurements in the magnetosheath [14]. The average magnitude of $\boldsymbol{V}$ was $115 \mathrm{~km} / \mathrm{s}$, about a factor of 4 smaller than the upstream solar wind speed. The direction of $\boldsymbol{V}$ was, however, $\sim 102^{\circ}$ from radial, indicating dawnward and sunward flow of the plasma in the expanding magnetosheath [14]. Using these parameters we represent the average downstream $\mathrm{H}^{+}$spectrum also as a sum of two CIKFs, shown by the bold solid curve. The measured portion of the downstream distribution is the strong tail that is well represented by a CIKF (dotted curve) with $n=$ $0.04 \mathrm{~cm}^{-3}, V=115 \mathrm{~km} / \mathrm{s}, V_{t h}=350 \mathrm{~km} / \mathrm{s}$ and $\kappa=2.9$. The only free parameters of the core portion of the $\mathrm{H}^{+}$spectrum (not measured) are the thermal speed and $\kappa$. The maximum $V_{t h}$ compatible with observations is $120 \mathrm{~km} / \mathrm{s}$.

In Fig. $4 \mathrm{~b}$ we show the pickup $\mathrm{He}^{+}$differential spectrum upstream and downstream of the shock. The upstream distribution has the expected cutoff at $\sim 20 \mathrm{keV} / \mathrm{e}$, corresponding to about twice the solar wind speed, and a weak tail. The core portion of the

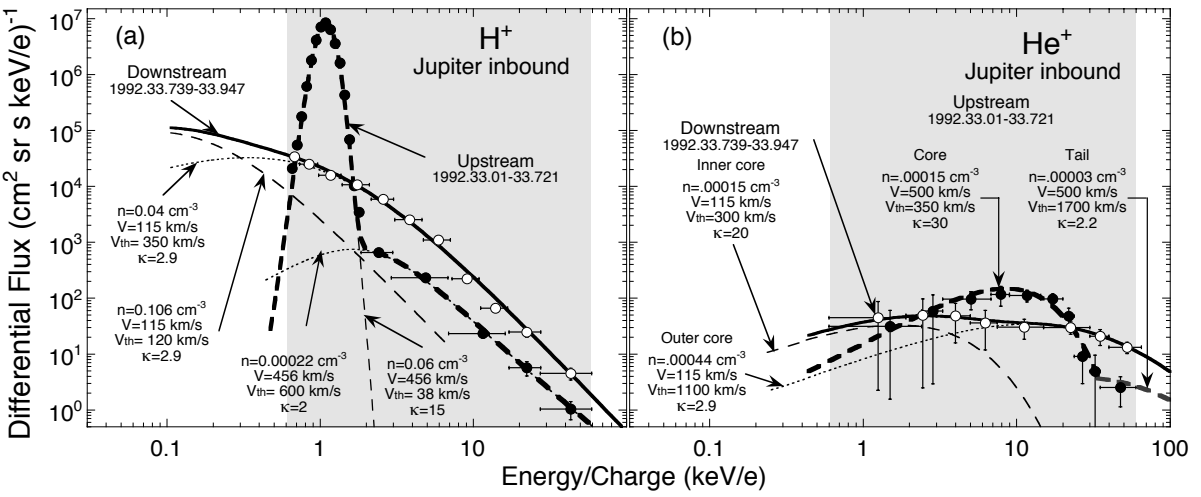

FIGURE 4. Differential intensity of (a) protons and (b) pickup $\mathrm{He}^{+}$upstream (filled circles) and downstream (open circles) of the Jovian bow shock, each averaged over the indicated time intervals. While the intensity of suprathermal $(\sim 2-60 \mathrm{keV} / \mathrm{e})$ protons downstream of the shock is $\sim 8$ times higher than upstream, the opposite is the case for $\mathrm{He}^{+}$whose downstream intensity between $\sim 2.5-25 \mathrm{keV} / \mathrm{e}$ is lower by $\sim 5$ compared to upstream, with no visible cutoff. Smooth curves are fits to the spectra using sums of convected isotropic kappa functions with parameters shown in the figure next to each curve. 
upstream $\mathrm{He}^{+}$spectrum is well represented by a CIKF (bold dashed curve) with $n=$ $0.00015 \mathrm{~cm}^{-3}, V=500 \mathrm{~km} / \mathrm{s}, V_{t h}=350 \mathrm{~km} / \mathrm{s}$ and $\kappa=30$. The tail portion is also fit with a CIKF with $n=0.00003 \mathrm{~cm}^{-3}, V=500 \mathrm{~km} / \mathrm{s}, V_{t h}=1700 \mathrm{~km} / \mathrm{s}$ and $\kappa=2.2$.

If we assume that the average measured compression factor of 2.43 also applies to $\mathrm{He}^{+}$, then the total density of the downstream $\mathrm{He}^{+}$spectrum should be $0.00044 \mathrm{~cm}^{-3}$. We represent the downstream $\mathrm{He}^{+}$distribution (open circles) with a CIKF using the measured average downstream convective velocity [14] and $n=0.00044 \mathrm{~cm}^{-3}$. The only free parameters are $V_{t h}$ and $\kappa$ which are determined to be $1100 \mathrm{~km} / \mathrm{s}$ and $2.9 \mathrm{re}-$ spectively by the best fit to the measured downstream $\mathrm{He}^{+}$spectrum above $\sim 5 \mathrm{keV} / \mathrm{e}$. At energies greater than $60 \mathrm{keV} / \mathrm{e}$ this fit is compatible with the differential total energy flux of ions [15] measured with the HI-SCALE instrument. Below $\sim 5 \mathrm{keV} / \mathrm{e}$ the CIKF fit is still within the error bars of the measured spectrum, although the addition of an 'inner core', shown as the light dashed curve, would fit the data best. This inner core contribution is most likely of local origin, namely $\mathrm{He}^{+}$ions escaping Jupiter's magnetosphere, or pickup $\mathrm{He}^{+}$produced in the magnetosheath from Jovian neutral He. The cutoff of this local Jovian pickup $\mathrm{He}^{+}$spectrum should be at the $\mathrm{He}^{+}$speed of $\sim 230 \mathrm{~km} / \mathrm{s}$, corresponding to the $\sim 4.5 \mathrm{keV} / \mathrm{e}$ cutoff of the inner core CIKF fit.

The solar wind electron temperature increased from $\sim 0.15 \mathrm{MK}$ to $\sim 1.2 \mathrm{MK}$ across the shock and then decreased almost linearly in the magnetosheath where the average temperature was $\sim 0.75 \mathrm{MK}[14]$. The corresponding average solar wind proton temperature, derived from the CIKF fit to the sheath core $\mathrm{H}^{+}$distribution, is $\sim 0.87 \mathrm{MK}$ $\left(V_{t h}=120 \mathrm{~km} / \mathrm{s}\right.$, see Fig. 4a). Assuming that the proton temperature decreased across the sheath at the same rate as the electron temperature, the $\mathrm{H}^{+}$temperature just downstream of the shock is $\sim 1.4 \mathrm{MK}$. The core $\mathrm{H}^{+}$temperature, $\sim 0.087 \mathrm{MK}\left(V_{t h}=38\right.$ $\mathrm{km} / \mathrm{s}$ ) upstream, jumps by a factor of $\sim 16$ at the shock. This agrees with the predicted temperature jump of $\sim 15.5$ computed from the shock compression ratio which is estimated to be $\sim 3.75$ from the change in the magnetic field strength $(\sim 0.67 \mathrm{nT}$ to $\sim 2.5 \mathrm{nT}$ [11]) and solar wind electron density $\left(\sim 0.05 \mathrm{~cm}^{-3}\right.$ to $\left.\sim 0.19 \mathrm{~cm}^{-3}[14]\right)$ across the shock. The corresponding temperature jump across the shock for pickup $\mathrm{He}^{+}$is $\sim 15.8$, while that for solar wind electrons is only $\sim 8$, or half as much as that for $\mathrm{H}^{+}$and $\mathrm{He}^{+}$.

The tail portions of the distributions seem to behave differently. For $\mathrm{H}^{+}$the fraction of the density in the tail jumps from $\sim 0.004$ upstream to $\sim 0.27$ in the magnetosheath, yet the thermal speed decreases by about a factor of 2 . This temperature decrease may be an artifact of the CIKF fits to the tails, since ions with energies greater than a few $\mathrm{MeV}$ are apparently not affected by this shock [15]. In the case of $\mathrm{He}^{+}$the entire core distribution appears to be heated, with no evidence of a distinct tail in the observed average magnetosheath spectrum.

\section{CONCLUDING REMARKS}

We concentrated here on observations of pickup $\mathrm{He}^{+}$and solar wind $\mathrm{H}^{+}$and $\mathrm{He}^{++}$upstream and downstream of the Jovian bow shock. We believe that these results have important implications for ion heating and acceleration as well as the production of 
ENAs at the termination shock. Not discussed were observations and studies of the ubiquitous suprathermal tails seen in the quiet and disturbed slow wind, as well as in the super-quiet fast wind from polar coronal holes [16]. Instead of diminishing rapidly with heliocentric distance, these tails are found to persist to at least 5.4 AU where they become even harder [16]. Also not discussed here (but see $[5,17,18]$ ) were chemical and isotopic abundances in the Local Interstellar Cloud deduced from observations of interstellar pickup ions with the SWICS instruments on Ulysses and ACE, as well as other interesting characteristics of interstellar and inner source pickup ions.

\section{ACKNOWLEDGMENTS}

We thank Ed Roelof and Lou Lanzerotti for describing HI-SCALE observations during 2 February 1992, Bob MacDowall for providing electron densities in the Jovian magnetosheath, and Christine Gloeckler for her help with data analysis and her constructive suggestions for improving the paper. This work was supported in part by NASA/JPL contract 955460 and NASA/Caltech grant NAG5-6912.

\section{REFERENCES}

1. Blum, P. W., and Fahr, H. J., Astrophys. Lett. 5, 127-130 (1970).

2. Axford, W. I., "The Interaction of the Solar Wind with the Interstellar Medium" in Solar Wind, edited by C. P. Sonett, P. J. Coleman, Jr., and J. M. Wilcox, NASA SP-308, Washington: National Aeronautics and Space Administration, 1972, pp. 609-658.

3. Möbius, E., Hovestadt, D., Klecker, B., et al., Nature 318, 426-429 (1985).

4. Gloeckler, G., Geiss, J., Balsiger, H., et al., Science 261, 70-73, (1993).

5. Gloeckler, G., Geiss, J., and Fisk, L. A., "Heliospheric and interstellar phenomena revealed from observations of pickup ions", in The Heliosphere near Solar Minimum: the Ulysses Perspectives, edited by A. Balogh, E. J. Smith, and R. G. Marsden, Springer-Praxis, Berlin, 2001, pp. 287-326.

6. Geiss, J., Gloeckler, G., Fisk, L. A., and von Steiger, R., J. Geophys. Res. 100, 23,373-23,377, (1995).

7. Gloeckler, G., Fisk, L. A., Geiss, J., Schwadron, N. A., and Zurbuchen, T. H., J. Geophys. Res. 105, 7,459-7,463 (2000).

8. Schwadron, N. A., and McComas, D. J., this Conference (2004).

9. Gloeckler, G., Geiss, J., Balsiger, H., et al., Astron. Astrophys. Suppl. Ser. 92, 267-289 (1992).

10. McComas, D. J., et al., this Conference (2004).

11. Balogh, A., Dougherty, M. K., Forsyth, R. J., et al., Science 257, 1515-1518 (1992).

12. Stone, R.G., Pedersen, B.-M., Harvey, C. C., et al., Science 257, 1524-1531, (1992).

13. MacDowall, R. J., private communication (2004).

14. Phillips, J. L., Bame, S. B., Thomsen, M. F., Goldstein, B. E., and Smith, E. J., Geophys. Res. 98, 21,189-21,202 (1993).

15. Roelof, E. C., private communication (2004).

16. Gloeckler, G., "Ubiquitous suprathermal tails on the solar wind and pickup ion distributions", in Solar Wind Ten: Proceedings of the Tenth International Solar Wind Conference, edited by M. Velli, R. Bruno, and F. Malara, AIP Conference Proceedings 679, New York: American Institute of Physics, 2003, pp. 583-588.

17. Gloeckler, G., Möbius, E., Geiss, J., et al., Astron. Astrophys., in press (2004).

18. Gloeckler, G., and Geiss, J., Advances Space Res., in press (2004).

19. Krimigis, S. M., Decker, R. B., Hill, M. E., et al., Nature 426, 45-48 (2003). 